\title{
Acute Limb Ischemia in One Dose Vaccination of COVID-19 Kemas Dahlan ${ }^{*}$, Yulianto Kusnadi ${ }^{2}$, Edo Tondas ${ }^{3}$, Daffa Faturrahman ${ }^{4}$
}

\begin{abstract}
Introduction: The extraordinary thrombotic manifestations of Corona virus Disease-2019 (COVID-19) caused by Severe Acute Respiratory Syndrome CoV-2 (SARS-CoV-2) virus, presenting as venous and arterial thrombosis have been reported in several literatures, but in this report, we presented a special case of acute limb ischemia in a patient that has been vaccinated by COVID-19 vaccine. Acute limb ischemia (ALI) is defined as decreasing in arterial perfusion of a limb with a threat to viability of the limb and mortality. COVID-19 has been declared as a global pandemic by the WHO. Patients with COVID-19 have abnormalities in blood coagulation parameters and are prone to thromboembolic events. This hypercoagulable state caused by COVID-19 mainly manifests as venous thromboembolism while peripheral arterial involvement is less frequent.
\end{abstract}

Case: A 54-year diabetic and smoker male has already got one dose COVID-19 vaccination came to our emergency unit with severe acute respiratory syndrome and pain, pale and paresthesia of his left leg. There was pneumonia pattern according to chest X-ray and a ground glass opacity in chest CT-scan, he was confirmed infected by COVID-19 according to RT-PCR test. From CT-Angiography we found multiple thrombosis in the distal aorta, superficial femoral artery (SFA), anterior tibial artery (ATA), and posterior tibial artery (PTA) of left lower extremity. Initial treatment we started with unfractionated heparin, oxygenation, intravenous fluid and analgesia. We managed the patient in isolation ward special for COVID-19. We did thrombectomy to save the limb, nevertheless in few days after we performed amputation at the level of talonavicular joint to the distal part of left foot caused by gangrene.

Conclusion: Vaccination can protect from COVID-19 if we vaccinated with full dose and our immune system able to create antibody. The condition will be different in immunocompromised condition and we get not full dose vaccination. Covid-19 Patient with Diabetes and smoker could have higher risk to develop acute limb ischemia because of hypercoagulable state. Management of a COVID-19 patient with cute limb ischemia is more complicated and challenging. Although we have already vaccinated life style modification like wearing a mask, social distancing and using hand sanitizer can give us more protection from Covid 19.

Keywords: acute limb ischemia, COVID-19 vaccination, heparinization, thrombectomy https://doi.org/10.36864/jinasvs.2021.2.001

*Correspondence: dokterdahlanspb@gmail.com

${ }^{1}$ M.D., Department of Surgery, Charitas Hospital, Palembang, Indonesia ${ }^{2}$ M.D., Department of Internal Medicine, Charitas Hospital, Palembang, Indonesia

3M.D., Department of Cardiology, Charitas Hospital, Palembang, Indonesia

${ }^{4}$ M.D., Faculty of Medicine, University of Sriwijaya, Palembang, Indonesia

\section{INTRODUCTION}

Since the spread of severe acute respiratory syndrome coronavirus (SARS-CoV-2) virus, various manifestations of this novel entity; Corona virus Disease-2019 (COVID-19) have been reported, including acute respiratory distress syndrome, cytokines storm, multi organ failure and notably, diffuse venous and arterial thrombosis. The degree of
Copyright (C) 2021, The Indonesian Society for Vascular and Endovascular Surgery

JINASVS 2021;2(2):1-4 widespread thrombosis reported in COVID-19 has not been previously seen with the SARS-CoV and Middle Eastern respiratory syndrome (MERS) in 2002 and 2012 [1]. Diffuse organ involvement in SARS-CoV-2 is likely due the virus high affinity to bind to the human angiotensin-converting enzyme 2 receptor (ACE 2) [2]. ACE-2 is widely expressed throughout the body, including the endothelium [3]. Direct viral infection and endothelial inflammation is hypothesized to result in the vascular pathologies seen in COVID-19 [3].

Acute limb ischemia (ALI) is defined as an obvious decrease in arterial perfusion of a limb with a threat to viability of the limb [4]. The clinical presentation is considered to be acute if symptom duration is less than 2 weeks [4]. The most common causes include embolism from cardiac chambers 
mainly associated with atrial fibrillation or acute myocardial infarction, embolism from arterial aneurysms, thrombosis of native limb arteries or vascular grafts, iatrogenic thromboembolism during vascular interventions, aortic dissection, and traumatic vascular injuries [4]. Corona virus disease 2019 (COVID-19) is caused by severe acute respiratory syndrome Corona virus 2 (SARS-CoV-2), and has been declared as a global pandemic by the World Health Organization [5]. Several reports revealed that patients with COVID-19 have abnormalities in blood coagulation parameters and are prone to thromboembolic events [5-6]. This hypercoagulable state caused by COVID-19 is associated with poor overall prognosis, and mainly manifests as venous thromboembolism (VTE) $[6,7]$. Peripheral arterial involvement is less frequent $[5,6]$. Herein we present a case of a spontaneous ALI in a COVID-19 patient. if not managed properly it will cause the patient to be amputated and even cause death due to sepsis. In this case we managed to manage ALI and COVID-19 simultaneously.

\section{CASE}

A 54-year man came to our emergency unit with pain, pale and paraesthesia of his left leg (Fig. 1 ). He was not able to flex his toes and ankle joint, however he was barely able to flex his knee joint. We felt a cool sensation from his distal toes until the ankle. He is a smoker and has diabetes without hypertension, has already got one dose COVID-19 vaccination about 3 weeks before admission. After got one dose vaccination the patient travel to his family and meet many people. At that time, he did not know that one dose vaccination not enough to prevent from COVID-19 infection, one week before the second dose vaccination he got cough and fever, he took medicine by himself but the cough and fever became more severe.

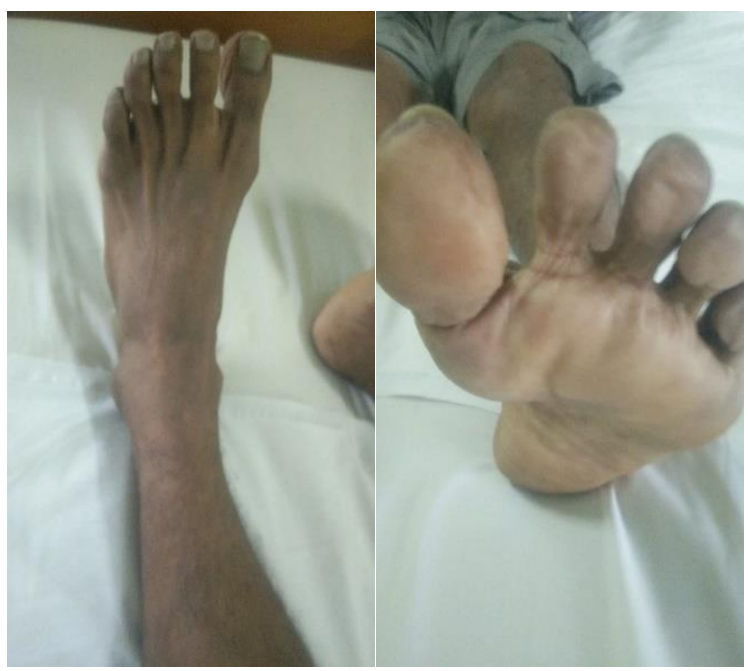

Figure 1a. Pale, cyanotic, pain, paraesthesia, and unable to move his ankle and left foot

From the chest X-ray (Fig. 3) we found an opacity appearance that we thought was a pneumonia. We suggested to do a CT thorax. We found ground glass opacity in the right and left lung (Fig. 4); the patient was COVID-19 confirmed according to PCR test. From electrocardiography there were no sign of arrythmia cordis nor ischemic, we performed echocardiography and there was no thrombus in ventricle and atrium also ejection fraction within normal range $72.8 \%$ (Fig.2). The patient had comorbidities with uncontrolled type 2 diabetes mellitus (DM) with HbA1C level in 9 and history of smoker. At that time, we got WBC $14.9 / \mathrm{mm}^{3}$, neutrophil $71 \%$, lymphocyte $15 \%$, ESR $46 \mathrm{~mm} / \mathrm{h}$ and D-dimer 4987. Then we diagnosed the patient with COVID-19 with Acute Limb Ischemia (ALI) Rutherford IIb, normo-weight uncontrolled DM.

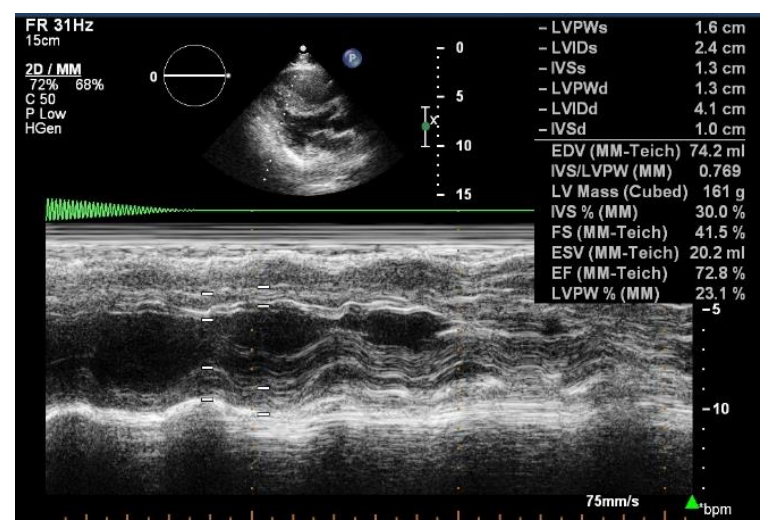

Figure 2. Echocardiography with ejection fraction $72.8 \%$ and there was no thrombus in the heart.

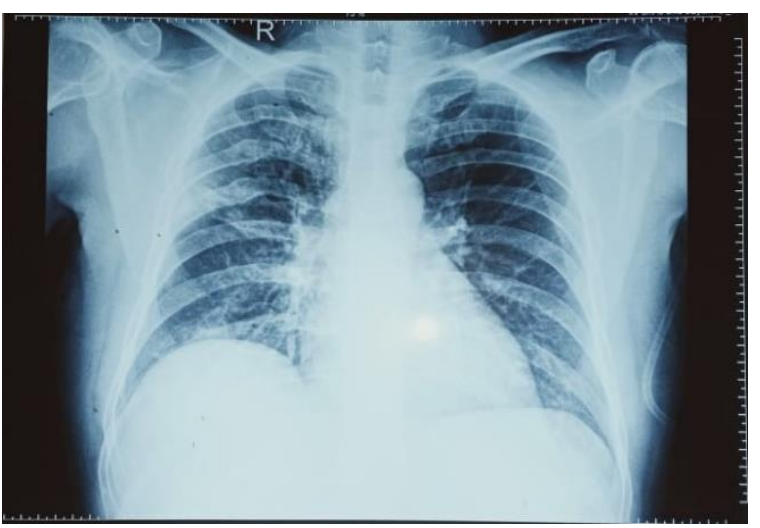

Figure 3. Chest X-ray showed radiopacity appearance in the upper and lower lobe of the right lung and basal of the left side

For the initial treatment in emergency department, we gave high molecular weight heparin and Ceftriaxone 1 gram every 12 hours, Ketorolac 30 mg every 12 hours, and Omeprazole $20 \mathrm{mg}$ twice daily, oxygen therapy, and intravenous rehydration. Treatment for diabetes and we used Levemir and Novorapid.

After we controlled the blood sugar below $200 \mathrm{mg} / \mathrm{dl}$ we revascularized the ischemic limb by thrombectomy. We did CT-angiography before and from the result we could see there were multiple thrombosis. There was thrombosis in the aortic bifurcation, iliac artery, femoral artery and popliteal artery with no atherosclerotic and minimal collateral that was mean the proses of thrombosis was acute event (Fig. 5,6)

We performed thrombectomy to the patient after waiting for more than 48 hours due to operating theatre queue, unfortunately in the condition of the distal leg more cyanotic and colder. We did thrombectomy for the thrombosis in the iliac artery, superficial artery, and tibio-peroneal trunk and 


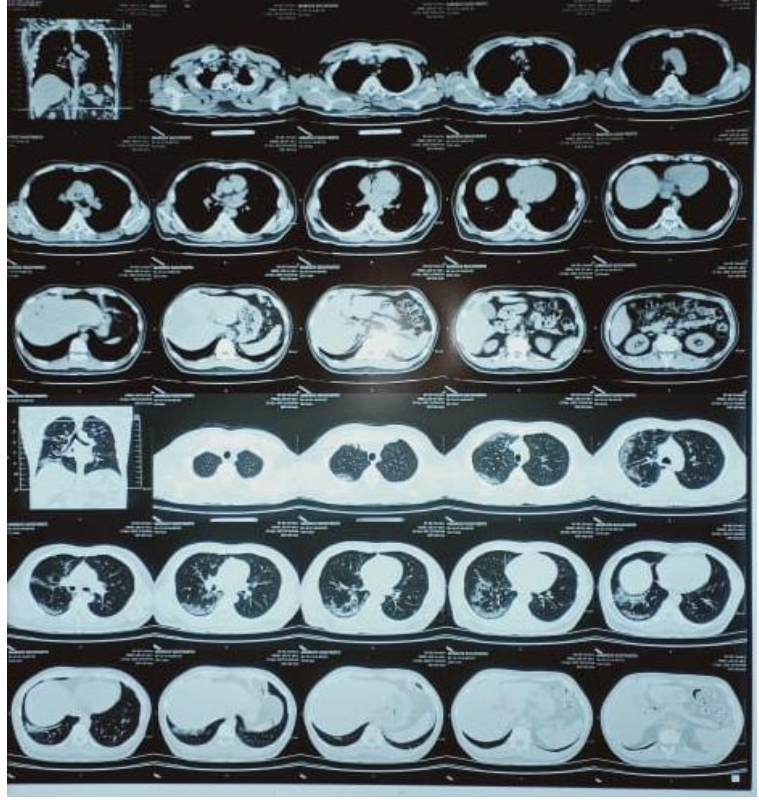

Figure 4. Ground glass appearance in the right and left lung

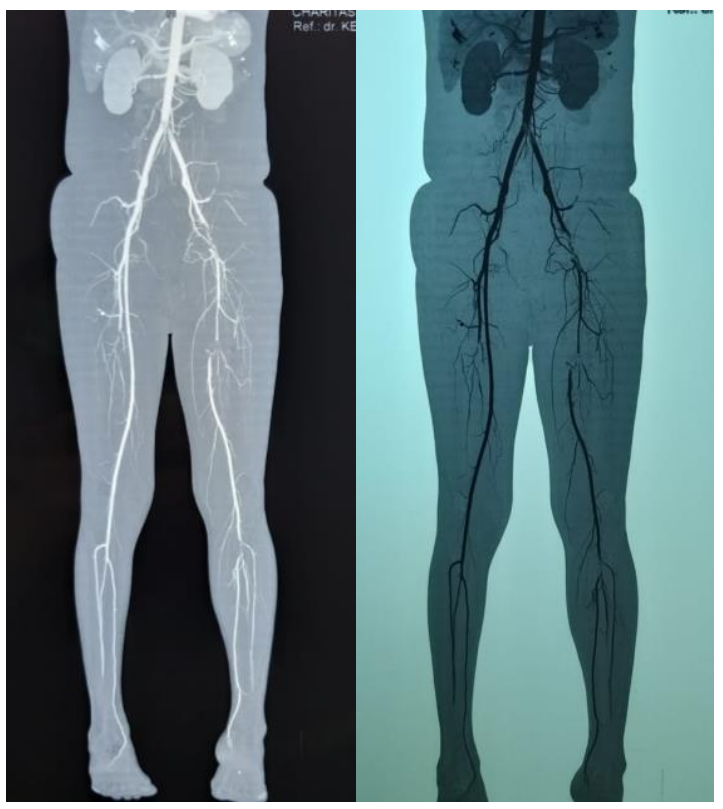

Figure 5. CT angiography showed thrombosis near the aortic bifurcation, common femoral artery, superficial femoral artery, and left tibioperoneal trunk

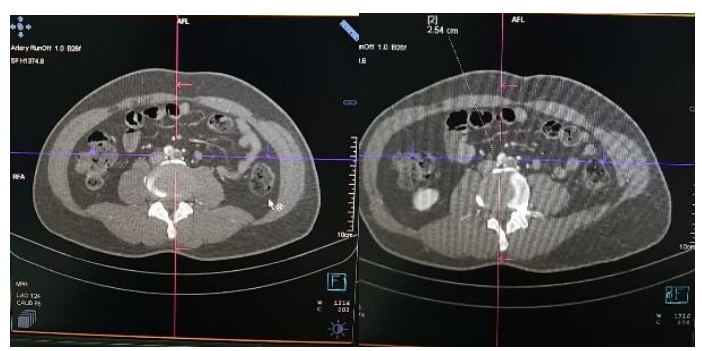

Figure 6. Thrombus in the bifurcation of aorta from CT angiography performed amputation at the level of talonavicular joint to eradicate the gangrene of the distal part of left foot (Fig. 7).

\section{DISCUSSION}

COVID-19 was declared a worldwide pandemic by the World Health Organization on March 11,2020 . While many patients develop mild-tomoderate symptoms (fever, fatigue, myalgia, headache, diarrhoea, dry cough, and respiratory distress), several patients have been described to have severe systemic disease, resulting in a myriad of coagulation abnormalities [8].

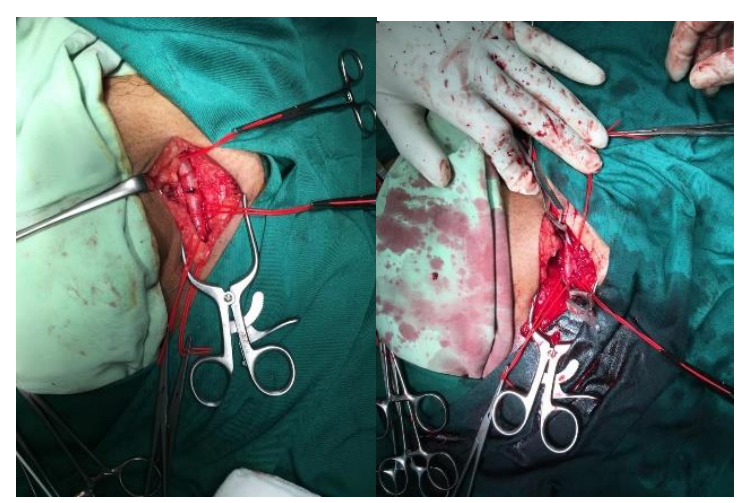

Figure 7. Thrombo-embolectomy from left femoral artery antegrade dan retrograde using Fogarty ${ }^{\circledR}$ catheter, we made a control incision in the right femoral artery to avoid distal embolism.

Angiotensin-converting enzyme 2 (ACE-2) receptors causes the systemic effect in COVID-19. They are expressed in several degrees in alveolar cells, endothelial cells, small intestinal cells, immune organs, etc [9]. After endothelial injury due to SARSCoV-2 attacks, they activate the coagulation cascade. In result are excessive cytokine release and storm, including activation of coagulation factors and meanwhile the fibrinolysis are also inhibited, resulting an extensive thrombosis. Interleukin- 6 is the key factor in COVID-19 thrombosis event. It upregulates the VEGF expression, then stimulates monocytes to express the tissue factors and resulting in extrinsic pathway of coagulation activation. A high d-dimers could be an indicator for higher mortality rate in patient with ischemia and thrombosis disease $[10,11,15]$.

The role of anticoagulants for prevention on thrombotic complications and to improve the overall prognosis in COVID-19 patients have not been clearly established. Tang et al. showed of 499 patients with COVID-19, out of which 99 patients received LMWH therapy, reported that anticoagulant therapy mainly with LMWH appears to be associated with better outcomes in severe COVID-19 patients [12]. It was quite interesting, that our patient has been treated with unfractionated heparin, his gangrene continued to worsen, ultimately requiring vascular intervention. The evidence for prophylactic anticoagulation for venous thromboembolism is still unclear, however prophylactic or full anticoagulation will be beneficial for arterial thrombosis caused by COVID-19 [13,15]. In current practice, patients with viable tissue and marginally threatened such as the Rutherford IIa or IIb could be performed vascular intervention for arterial thrombolysis (CDT-Catheter Directed 
Thrombolysis) and/or thrombectomy [14]. Limbs with irreversible ischemia require amputation, as in our clinical case.

\section{CONCLUSION}

Vaccination can protect from COVID-19 if vaccinated with full dose and our immune system able to create antibody. The condition will be different in immune compromised condition and not full-dose vaccination. COVID-19 patient with diabetes and smoker could have higher risk to develop acute limb ischemia because of hypercoagulable state condition. Management of a COVID-19 patient with cute limb ischemia is more complicated and challenging. Wearing a mask, social distancing and using hand sanitizer can reduce the risk for being infected by COVID-19.

\section{CONFLICT OF INTEREST}

The author states the original work, and there is no conflict of interest in doing this research.

\section{ORCID ID OF AUTHORS}

Kemas Dahlan

https://orcid.org/0000-0002-0153-4384

Yulianto Kusnadi

Edo Tondas

Daffa Faturrahman

\section{REFERENCES}

1. Miesbach, Wolfgang, and Michael Makris. "COVID-19: coagulopathy, risk of thrombosis, and the rationale for anticoagulation." Clinical and Applied Thrombosis/Hemostasis 26 (2020): 1076029620938149.

2. Kariyanna, Pramod Theetha, et al. "A Systematic Review of COVID-19 and Myocarditis." American journal of medical case reports 8.9 (2020): 299.

3. Varga, Zsuzsanna, et al. "Endothelial cell infection and endotheliitis in COVID-19." The Lancet 395.10234 (2020): 1417-1418.

4. M. Bjorck, J.J. Earnshaw, S. Acosta, F.B. Goncalves, C. Frederic, E.S. Debus, , R. Hinchliffe, V. Jongkind, M.J.W. Koelemay, G. Menyhei, A.V. Svetlikov, Y. Tshomba, J.C. Van Den Berg, Editor's choice-European society for vascular surgery (ESVS) 2020 clinical practice guidelines on the management of acute limb ischaemia, Eur. J. Vasc. Endovasc. Surg. 59 (2) (2020) 173-218.

5. D. Gomez-Arbelaez, G. Ibarra-Sanchez, A. Garcia-Gutierrez, A. Comanges-Yeboles, M. AnsuateguiVicente, J.A. Gonzalez-Fajardo, COVID-19-related aortic thrombosis: a report of four cases, Ann. Vasc. Surg. 67 (2020) 10-13.

6. S. Bilaloglu, Y. Aphinyanaphongs, S. Jones, E. Iturrate, J. Hochman, J.S. Berger, Thrombosis in hospitalized patients with COVID-19 in a New York city Health system, JAMA 324 (8) (2020) 799-801.

7. N. Tang, D. Li, X. Wang, Z. Sun, Abnormal coagulation parameters are associated with poor prognosis in patients with novel Corona virus pneumonia, J. Thromb. Haemostasis 18 (4) (2020) 844-847.

8. Levi M, Thachil J, Iba T, Levy JH: Coagulation abnormalities and thrombosis in patients with COVID-19. Lancet Haematol. 2020, 7:438-440. 10.1016/S2352-3026(20)30145-9.

9. Wan Y, Shang J, Graham R, Baric RS, Li F: Receptor recognition by the novel Corona virus from Wuhan: an analysis based on decade-long structural studies of SARS Corona virus. J Virol. 2020, 94:e00127-20. 10.1128/JVI.00127-20.

10. Zhou $Y$, Fu B, Zheng $X$, et al.: Aberrant pathogenic GM-CSF+ T cells and inflammatory CD14+ CD16+ monocytes in severe pulmonary syndrome patients of a new Corona virus [PREPRINT]. BioRxiv (ed): 10.1101/2020.02.12.945576.

11. Zhou F, Yu T, Du R, et al.: Clinical course and risk factors for mortality of adult inpatients with COVID-19 in Wuhan, China: a retrospective cohort study. Lancet. 2020, 395:1054-1062. 10.1016/S01406736(20)30566-3.

12. Tang N, Bai H, Chen X, Gong J, Li D, Sun Z: Anticoagulant treatment is associated with decreased mortality in severe Corona virus disease 2019 patients with coagulopathy. J Thromb Haemost. 2020, 18:1094-1099. 10.1111/jth.14817.

13. Connors JM, Levy JH: COVID-19 and its implications for thrombosis and anticoagulation. Blood. 2020, 135:2033-2040. 10.1182/blood.2020006000.

14. Anton N. Sidawy, Bruce A. Perler, et al: Rutherford's Vascular Surgery and Endovascular Therapy

15. Suhartono R, Nugroho NT. Dealing with hypercoagulability problem in COVID-19 cases. Journal of Indonesian Soc of Vasc and Endovasc Surg. doi.org/10.36864/jinasvs.2021.1.001 